\title{
TRIPLICIDAD URETERAL. UNA INUSUAL PRESENTACIÓN AISLADA
}

\author{
A. VILLANUEVA PEÑA, E. DE DIEGO RODRÍGUEZ \\ Servicio de Urología. Hospital Comarcal de Laredo. Cantabria
}

Actas Urol Esp. 28 (10): 766-770, 2004

\section{RESUMEN}

TRIPLICIDAD URETERAL. UNA INUSUAL PRESENTACIÓN AISLADA

Presentamos el caso de una paciente de 26 años que presenta como hallazgo casual una triplicidad ureteral derecha (probablemente tipo I de Campbell), sin ninguna otra alteración asociada.

Pese a que la duplicidad ureteral es una alteración urológica frecuente, la existencia de tres uréteres en un mismo lado, ya sea de forma completa o incompleta, representa una verdadera rareza, con menos de cien casos recogidos en la literatura.

$\mathrm{El}$ presente caso resulta singular no sólo por lo excepcional de esta entidad, sino por no encontrarse asociado a ninguna otra alteración urológica o extraurológica ni presentar manifestación clínica alguna.

Se realiza una revisión y actualización de esta entidad.

PALABRAS CLAVE: Uréter. Triplicidad ureteral.

\section{ABSTRACT}

\section{URETERAL TRIPLICATION, AN UNUSUAL ISOLATED PRESENTATION}

We report the case of a 26 years old female who presented, as a casual finding, a right complete ureteral triplicity ( probably Campbell's tipe I) with no other associated abnormalities.

Despite ureteral duplication is a frequent urological anomaly, the presence of three ureters occurring in the same side, in a complete or incomplete form, is a true rarity, with less than 100 cases publicated up to date.

The present case is singular, not only because of its exceptional presentation but also because no other urological or extraurological anomalies were associated and no clinical manifestations were present.

The most relevant literature is reviewed.

KEY WORDS: Ureter. Ureteral triplication.

$\mathrm{L}^{2}$

a existencia de tres uréteres en un mismo lado drenando una única unidad renal resulta una hallazgo extremadamente raro, con menos de 100 casos recogidos en la literatura. Esta anomalía se encuentra, por norma, asociada a otras diversas alteraciones congénitas, responsables en la mayor parte de los casos de manifestaciones clínicas precoces (infección, dolor, incontinencia...). No obstante, la triplicidad ureteral ha sido descrita también en pacientes de mayor edad como hallazgo fortuito en estudios por procesos no relacionados.

La coincidencia de ambas circunstancias (ausencia de otras anomalías asociadas y curso asintomático) representa una verdadera curiosidad. 


\section{CASO CLÍNICO}

Se trata de una paciente de 26 años asistida en la consulta de ginecología por dolor menstrual. En la ecografía renal solicitada presentaba una aparente ectasia del grupo superior del riñón derecho, por lo que ante la sospecha de duplicidad ureteral con dilatación del hemirriñón superior, es remitida a nuestra consulta para valoración urológica.

La paciente no presentaba antecedentes urológicos de interés ( infección urinaria, incontinencia, hematuria y/o dolor lumbar),y la exploración física no aportó datos de interés.

La analítica sanguínea (hemograma y bioquímica) y urinaria (elemental y sedimento y cultivo) eran normales

El estudio urográfico puso de manifiesto la existencia de una triplicidad ureteral en el lado derecho (probablemente tipo I de Campbell) y riñón contralateral normal con un único sistema excretor. Ambas unidades renales presentaban buen funcionamiento bilateral, sin signos de ectasia (Figs. 1 y 2) y la cistografía no mostraba la existencia de reflujo en ninguna de las unidades vesicoureterales (Fig. 3).

Dada la ausencia de clínica y anomalías urológicas asociadas, la paciente rechazó la realización de estudio endoscópico.

Esta inconveniencia nos impide, desgraciadamente, poder definir con exactitud el tipo de triplicidad ureteral del que se trata, y aunque aparentemente los tres uréteres entran de forma independiente en la vejiga, no se puede descartar

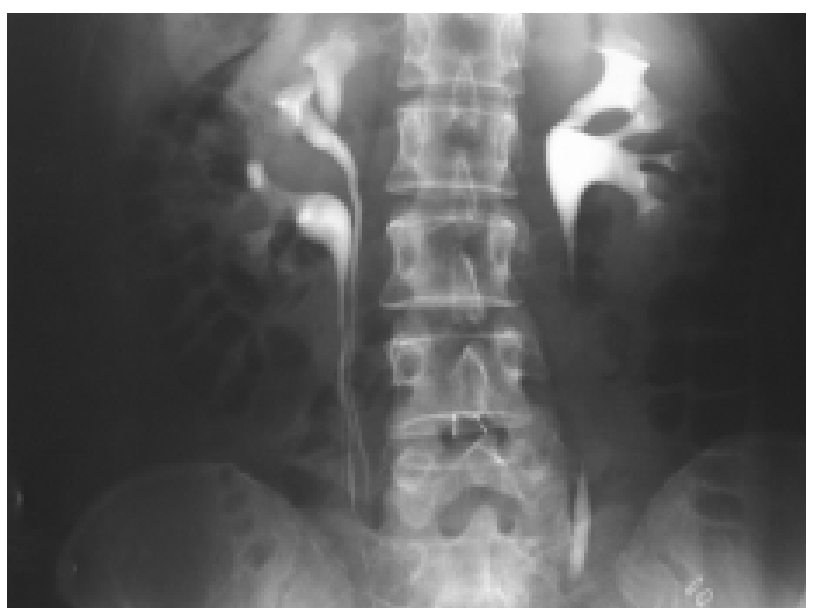

FIGURA 1. Triplicidad ureteral derecha. Uréter único izquierdo.

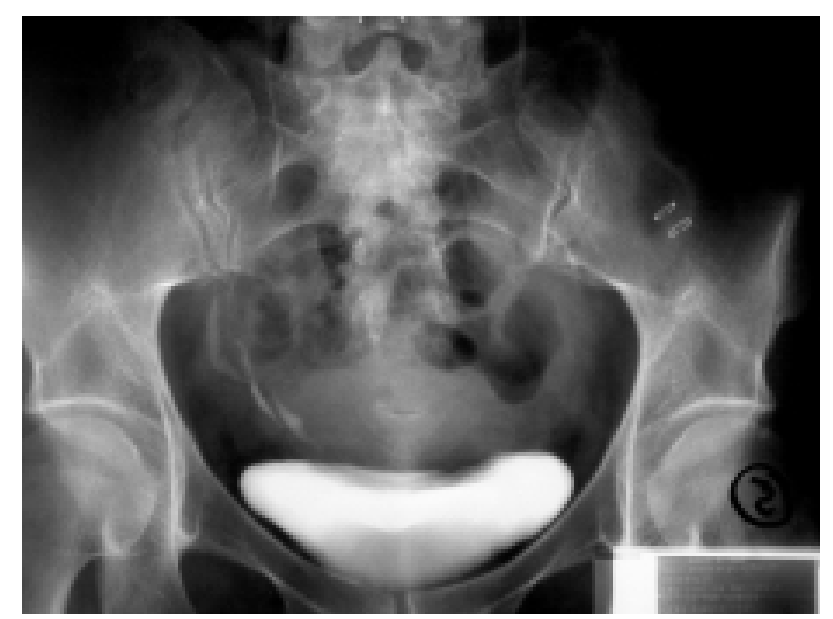

FIGURA 2. Detalle del triple uréter distal.

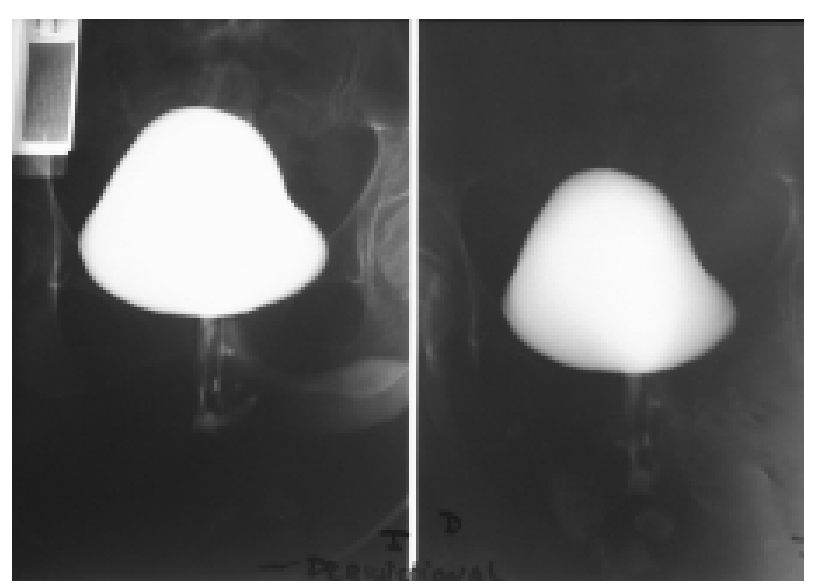

FIGURA 3. Ausencia de reflujo.

categóricamente una fusión distal de alguno de ellos. No obstante, la ausencia de clínica manifiesta o de signos radiológicos típicos que sugieran tal fusión (see-saw o efecto yo-yo) y la frecuencia de presentación nos inclinan a pensar que se trata de una triplicidad tipo I de Campbell.

\section{DISCUSIÓN}

Si consideramos básicamente la duplicidad ureteral, las alteraciones de número representan las alteraciones ureterales más frecuentes.

No existen series lo suficientemente amplias para establecer con exactitud la incidencia de dicha alteración, aunque se puede realizar una aproximación más o menos cercana a la realidad basándose en la experiencia acumulada en series clínicas (con un índice significativamente más alto) y procedente de necropsias, obteniéndose una incidencia en torno a $0,7 \%$ de la población ${ }^{1,2}$. 
Sin embargo, el resto de alteraciones numéricas ureterales resultan excepcionales, con una centena de casos recogidos de triplicidad ureteral y cuatro de uréter cuádruple ${ }^{3}$, la mayor parte de los cuales asocia algún otro tipo de alteración urológica morfológica y/o funcional con traducción clínica y compromiso, en gran parte de los casos, de alguno de los segmentos renales.

Para entender en toda su extensión la disposición anatómica de las unidades ureterales múltiples y las implicaciones funcionales que puede acarrear esta anomalía es necesario recurrir a la ayuda de la embriología.

El conducto mesonéfrico de Wolff desciende desde los somitas cervicales hasta hacer contacto con el seno urogenital hacia el día 28, produciéndose una fusión entre el epitelio del seno urogenital (endodermo) y el del conducto mesonéfrico (mesodermo). Una vez que el conducto drena en el seno urogenital, se origina del mismo el brote ureteral como una evaginación de la superficie posteromedial en la zona en la que se acoda para dirigirse hacia la cloaca.

El segmento del conducto mesonéfrico distal al origen del brote ureteral recibe el nombre de conducto excretor común ${ }^{4}$, que tras dilatarse dará lugar al hemitrígono correspondiente.

A medida que el conducto excretor común es absorbido en el seno urogenital, el brote ureteral y el conducto mesonéfrico adquieren orificios separados, localizándose inicialmente el uréter en una posición medial y distal. Más tarde el orificio del conducto mesonéfrico se desplaza en sentido caudal, de forma que el mesodermo de los conductos excretores dará origen al esqueleto del trígono primitivo, mientras que el uréter primitivo experimenta una migración craneal.

Cualquier alteración en la posición del brote ureteral tendrá evidentes repercusiones en la ubicación del uréter definitivo, de forma que cuando surge demasiado distal en el conducto mesonéfrico experimentará un ascenso mayor de lo habitual presentando una disposición más craneal y lateral respecto al trígono, con mayor probabilidad de presentar reflujo dado que el aporte de mesénquima al desarrollo muscular del trígono es menor en un conducto común corto. Puesto que además hay menos tiempo para el acúmulo de mesénquima alrededor del brote ureteral, la parte intramural del uréter tiene también una musculatura deficiente.

Cuando la yema ureteral surge en una posición especialmente alta en el conducto de Wolff, migrará caudalmente durante un lapso de tiempo mayor unido al conducto mesonéfrico antes de separarse, pudiendo aparecer distalmente en un trayecto predecible denominado vía ectópica ${ }^{5} \mathrm{o}$ incluso podrá conservar su unión con el conducto mesonéfrico.

En aquellas circunstancias en las que se produzcan brotaciones ureterales supernumerarias, se originarán anomalías ureterales de número. El caso más frecuente es la duplicidad ureteral. En fases muy tempranas, cuando ambos uréteres son absorbidos en el trígono, el brote más cercano al seno urogenital dará lugar al uréter del hemirriñón inferior. El segundo brote, que dará lugar al uréter del hemirrinón superior, es absorbido más tardíamente, migrando y rotando en torno al orificio del uréter inferior acompañando al conducto mesonéfrico para acabar en una posición distal y medial respecto a aquél (ley de Weigert Meyer). Stephens ${ }^{5}$ basándose en los postulados de Ireland ${ }^{6}$ ha propuesto una teoría para explicar los raros casos de duplicidad que excepcionalmente no cumplen esta norma, de manera que el orificio del uréter del hemirriñón superior tendría su origen en un brote ureteral unional (bifurcación muy precoz) en lugar de un segundo brote.

De igual forma, la aparición de tres brotes ureterales o bien la división temprana de una de dos yemas ureterales dará lugar a triplicidades completas o incompletas respectivamente. No obstante, en estos casos no es tan predecible el cumplimiento de la ley de Weigert Meyer ${ }^{5}$.

La clasificación más utilizada en las triplicidades ureterales es la desarrollada por Smith ${ }^{7}$ que las agrupa en cuatro categorías (Fig. 3):

Tipo I. Triplicidad completa. Tres uréteres desde el rinón, con tres orificios de drenaje independientes. Esta parece ser la forma de presentación más fruecuente $(35 \%)^{8}$.

Tipo II. Triplicidad incompleta. Tres uréteres desde el riñón, con un uréter bífido y dos orificios de drenaje (21\%). 
Tipo III. Uréter trífido. Tres uréteres desde el riñón con un único orificio de drenaje (31\%).

Tipo IV. Dos uréteres desde el riñón, con una bifurcación invertida en uno de ellos y tres orificios de drenaje.

La triplicidad ureteral es una anomalía rara, habitualmente asociada a otras alteraciones congénitas con traducción clínica o no, lo que puede provocar que su diagnóstico sea fortuito. La posible ausencia de signos clínicos podría explicar que dicha malformación pase desapercibida, especialmente cuando está asociada a segmentos renales displásicos ${ }^{9}$ lo que justificaría el amplio rango de edad al diagnóstico recogido en la literatura de 1 a 74 años ${ }^{10}$.

La anomalía más frecuentemente asociada a la triplicidad ureteral es la duplicidad contralateral $(37 \%)^{10-17}$, la ectopia ureteral $(28 \%)^{14,15,18-21}$ o la displasia renal ${ }^{9,22,23}$ y ocasionalmente puede estar asociada con síndromes polimalformati$\operatorname{vos}^{24-26}$. La existencia de ureterocele o uréter ectópico está bien documentado en la duplicidad ureteral y de igual forma sucede en la triplicidad $^{9,17,23,27-30}$, especialmente aquél último, lo que traduce su frecuente asociación con el reflujo vesicoureteral ${ }^{11,12,17,23,29,31-33}$ y la obstrucción ${ }^{22,33}$.

Por otro lado, se han descrito formas excepcionales de presentación en riñón único ${ }^{31}$, en ectopia renal cruzada $^{34}$, riñón en herradura ${ }^{35} \mathrm{o}$ formas bilaterales ${ }^{34,36}$ que no dejan de ser comunicaciones anecdóticas dentro del amplio espectro de alteraciones asociadas.

Cuando la triplicidad ureteral presenta manifestaciones clínicas, éstas resultan inespecíficas (lumbalgia, fiebre, síndrome miccional, incontinencia) y están en relación con las alteraciones asociadas (reflujo, obstrucción, ectopia...). Son precisamente esas alteraciones las que determinarán la oportunidad de cada opción terapéutica individualizada (nefrectomía parcial o total y ureterectomía, resección de ureterocele, urétero o pieloureterostomía o reimplantación ureteral).

De forma eventual, como sucede en este caso, la UIV puede ser diagnóstica, pero en la mayor parte de las ocasiones, especialmente en los asociados a otras alteraciones como ectopia o displasia renal segmentaria puede ser necesaria una importante batería de pruebas diagnósticas para su reconocimiento (cistografía, cistoscopia, pielografía retrógrada, TAC o pruebas isotópicas) ${ }^{10}$.

En la revisión realizada, las triplicidades ureterales tipo I como la aquí presentada, estaban asociadas a alguna otra alteración, como reflujo $^{11,23,31,32}$ o ectopia ureteral ${ }^{19,20}$ y sus correspondientes repercusiones clínicas y/o morfofuncionales sobre las unidades renales. El presente caso resulta una rara excepción a la norma incluyendo todos los tipos de ureteral y tan sólo hemos encontrado otro recogido en la literatura que como el nuestro no presentaba manifestaciones clínicas ni ninguna otra alteración urológica asociada $^{8}$. En estas circunstancias, obviamente, no está justificada ninguna otra actitud que no sea la expectante.

\section{REFERENCIAS}

1. Engelstein D, Livne P, Maya C, et al. Type II ureteral triplication asociated with ectopic ureter. Urology 1996;48:786788.

2. Campbell MF. Anomalies of the ureter. En Campbell and Harrison J.H. Urology $3^{\text {rd }}$ ed. Philadelphia, W.B Saunders CO, 1970.

3. Sourtzis S, Damry N, Janssen F, et al. Ureteral quadriplication: the fourth case report. Pediatr Radiol 1994;24:604-605.

4. Alcaraz A, Vinaixa F, Tejedo-Mateu A, et al. Congenital obstructive disease of the ureter. Acta Urol Esp 1989;13: 318.

5. Stephens FD. Anatomical variages of double ureters. Aust N.Z. J Surg 1958;28:27.

6. Ireland EF, Chute R. A case of triplicate-duplicate ureters. J Urol 1955;74:342-347.

7. Smith I. Triplicate ureter. Br J Surg 1946;34:182-185.

8. Shingh G, Murray K. Ureteral triplication, occasionlly an isolated anomaly. Urol Int 1996;56:117-118.

9. Verdú F, Moncada I, Díez J, et al. Ureteral triplicity: trifid ureter with ipsilateral ureterocele and renal dysplasia in an adult with testicular seminoma. Actas Urol Esp 1990; 14:452-454.

10. Sánchez de Badajoz E, Ramos J, Burgos R. Ureteral triplication with contralateral ureter duplication Urol Int 1992; 48:217-218

11. Tarrado X, Rodo J, Morales L. Ureteral triplication with vesicoureteral reflux and contralateral duplication. Actas Urol Esp 2000;24:429-432.

12. Cherasse A, Marievoet C. Ureteral triplication and contralateral ureteral duplication. Apropos of a case. Acta Urol Belg 1997;65:35-41.

13. Srivastava DN, Mukhopadhayay S, Agarwal S. Ureteral triplication with contralateral duplication. Nephron 1996;74: 427-428.

14. Prantl M, Tewes G. Ureter triplication with contralateral ureter duplication. (abstract). Z Kinderchir 1998;43:350-352.

15. Gotoh S, Matsumura T, Kobayashi T, et al. A case of right triplicate and left duplicate ureters. (abstract). Hinyokika Kiyo 1987;33:1420-1422. 
16. Youngson GG. Ureteral triplication, contralateral duplication and bilateral extravesical ectopic ureter. $\mathrm{J}$ Urol $1985 ; 134: 533-535$.

17. Miguelez C, Gaztambide J, Camacho F, et al. Ureteral triplication and duplicated opposite kidney with refluxing ureterocele. J Pediatr Surg 1983;18:614-616.

18. Patel PM, Stock JA, Hanna MK, et al. Ureteral triplication with ectopic upper pole moiety. Urology 2001;58:279-280.

19. Luque-Mialdea R, De Tomas E, Arrojo F, et al. Ureteral triplicaton: double extravesical ureteral ectopia. J Urol 1991; 145:109-111.

20. Zaontz MR, Maizels M. Type I ureteral triplication: an extension of the Weigert Meyer law. J Urol 1985;134:949-950.

21. Bloom RA, Crooks KK, Wise HA. Complete ureteral triplication with ectopia. Urology 1985;25:176-178.

22. Bouhafs A, Dubois R, Chaffange $P$, et al. Two rare case reports of ureteral triplication. Ann Urol (Paris) 2002;36:4244.

23. Wilhelm E. A case of ureteral triplication associated with renal dysmorphy, bilateral reflux, ureterocele and AskUpmark kidney (abstract). Urologe A 1983;22:267-269.

24. Sánchez de Badajoz. Triplicación ureteral y duplicación de vejiga. Arch Esp Urol 1982;135:139-142.

25. Golomb J, Ehrlich R. VACTREL syndrome. J Urol 1989; 141:1398-1399.

26. Rich M, Heimler A, Waber L, et al. Autosomal dominant transmission of ureteral triplication and bilateral amastia. J Urol 1987;137:102-105.

27. Arap S, Lopes R, Mitre A, et al. Triplicite uréterale compléte, associée á une uréterocéle ectopique. J d'Urol 1982;88:167.
28. Juskiewenski S, Soulie M, Baunin C, et al. Ureteral triplication. Chir Pediatr 1987;28:314-316.

29. Rodo J, Bishara F, Claret I. Ureteral triplication with reflux and ureterocele. Arch Esp Urol 1986;39:343-347.

30. Finkel LI, Watts F, Corbett D. Ureteral triplication with a ureterocele. Pediatr Radiol 1983;13:346-348.

31. Ander H, Ziylan O, Cayan S, et al. A case of ureteral triplication (type I) associated with vesicoureteral reflux in a solitary kidney. Int Urol Nephrol 1997;29:537-540.

32. Hassan MA. Ureteral triplication (type I) with vesicoureteral reflux. Urology 1990;35:78-80.

33. Blumberg N. Ureteral triplication. J Pediatr Surg 1976;11: 579-580.

34. Golomb J, Ehrlich RM. Bilateral ureteral triplication with crossed ectopic fused kidneys associated with the VACTREL syndrome. J Urol 1989;141:1398-1399.

35. Pode D, Shapiro A, Lebensart P. Unilateral triplication of the collecting system in a horseshoe kidney. J Urol 1983; 130:533-534.

36. Shoda R, Ejiri S, Fujita T, et al. A case of bilateral ureteral triplication. (abstract) Hinyokika Kiyo. 1985;31:475-581.

\author{
Dr. A. Villanueva Peña \\ Calle Vargas $47,5^{\circ}$ puerta 6
}

30910. Santander.

(Trabajo recibido el 23 junio de 2003) 